\title{
Experiences, knowledge of functions, and social acceptance of robots: an exploratory case study focusing on Japan
}

\author{
Tatsuya Nomura ${ }^{1} \cdot$ Motoharu Tanaka $^{1}$
}

Received: 5 August 2020 / Accepted: 18 March 2021 / Published online: 29 March 2021

(c) The Author(s) 2021

\begin{abstract}
Although Japanese society has become aware of some types of robots, social acceptance of robots is still not widespread. This study conducted an online questionnaire survey to investigate the relationships between experiences with and knowledge of vacuum, pet-type, and communication robots and acceptance of these robots, including the intention to use and trust. The results suggested that experiences with, knowledge of functions, and acceptance of the robots differed depending on the type of robot, and the influence of these factors on acceptance of the robots also differed depending on the robot types.
\end{abstract}

Keywords Social acceptance $\cdot$ Experience $\cdot$ Knowledge $\cdot$ Type of robot

\section{Introduction}

2015, the government of Japan announced the "New Robot Strategy" and an action plan for Japan's economic revitalization, making it the first county to encourage the widespread use of robots (Prime Minister of Japan and His Cabinet 2015). This strategy consists of three pillars of the robot revolution, one of which is a realization of daily life with robots in the fields of manufacturing not only by large companies, but also major small and medium-sized companies, nursing and medical care, infrastructure, and disaster preparedness, and the service, construction, and agriculture industries. On the other hand, an online survey conducted in 2015 by Nomura Research Institute in Japan (Nitto et al. 2017) suggested that compared with the USA and Germany, Japanese society did not necessarily have more experience with or higher acceptance of robots. Now, 5 years later, it can be estimated that some types of robots have become more well-known in Japanese society.

To what extent robots, in general, are socially accepted in Japan has been investigated by some existing studies. Kochigami et al. (2018) revealed four types of robot

Tatsuya Nomura

nomura@rins.ryukoku.ac.jp

Motoharu Tanaka

T160384@mail.ryukoku.ac.jp

1 Department of Media Informatics, Ryukoku University, 1-5 Yokotani, Seta-Ohe-Cho, Otsu, Shiga 520-2194, Japan
Japanese people preferred (a robot doing housework, a communicating robot, a healthcare robot, and a pet robot) and age difference in acceptance of these robots. However, the sample number of their survey was small $(N=56)$. Lee et al. (2016) conducted a comparison study between Japan and South Korea ( $N=80$ for each nation) on evaluation for biologically inspired robots such as a humanoid robot and functionally designed robots such as a vacuum robot. Although their study found a cultural difference meaning that Japanese people more positively evaluated a humanoid robot than a vacuum robot and Korean people had the opposite trend, it was not investigated what factor influenced these evaluations. Bröhl et al. (2019) proposed a psychological model on social acceptance of robots based on the original technology acceptance model [TAM (Davis 1989)], and conducted an international online survey $(N=322$ from Germany, $N=360$ from Japan, $N=349$ from China and $N=295$ from the USA). Although their results suggested a high habituation effect and the immense spread of robots in everyday life as in restaurants and hotels in Japan, it was not investigated what type of robots were actually accepted. Although the white paper from the government of Japan in 2019 (Ministry of Internal Affairs and Communication Japan 2019) mentioned that a relatively high percentage of Japan's population had a positive image of humanoid robots compared to the United States and Germany, no concrete data was presented in the paper (although the Japanese version of the white paper mentioned the above 
research by Nomura Research Institute (Nitto et al. 2017) and another research report (Morikawa 2017), no data was shown about what type of robots was accepted in the current state of Japan). In this situation, it is important to clarify the types of robots currently accepted in Japan and the factors influencing this acceptance.

On the other hand, many existing studies have revealed influential factors into social acceptance of robots in addition to the ones mentioned above such as age and culture. The survey conducted by Reich and Eyssel (2013), of which participants were 366 German people, found that human gender, occupation, interest in science and technology, and dispositional correlates of anthropomorphism had a significant impact on attitudes towards service robots in domestic environments. There are also other existing studies suggesting the influence of anthropomorphism (Duffy 2003; Epley et al. 2007; Spatola et al. 2019a). Spatora et al. (2019b) conducted an online survey for 139 French people and revealed the when robots' countries of origin were presented, the stereotypes for the countries on warmth and competence affected the perception of the robots. Latikka et al. (2019) suggested that selfefficacy in using robots was associated with the acceptance to use humanoid, pet, and telepresence robots in the survey in which 3800 care work staffs participated. Smarr et al. (2012) revealed in their survey $(N=21)$ consisting of questionnaires and interviews that older adults' acceptance of robot assistance varied dependent on the sorts of tasks the robots performed. In the user study conducted by De Graaf and Allouch (2013) $(N=80)$, a lot of factors based on TAM such as enjoyment and anxiety were considered. Considering these factors, it is crucial how to focus on specific ones.

The study focuses on human experiences with robots. Some existing studies suggest that actual experiences with robots operating in the same physical space decreased negative attitudes toward robots (Nomura et al. 2006; Nomura 2014; Weiss et al. 2009). However, these studies did not consider types of robots, or a single type of robots (humanoid). It can be guessed that negative attitudes toward a specific type of robot may have decreased in current Japanese society since people have continuously been exposed to information via advertisements and real experiences with the robot in daily life. However, it should be clarified what type of robots have been actually experienced, as well as people's expectations, level of trust, and whether experiences with and knowledge of a robot actually affect social acceptance of the robot, and this affection differs between types of robots.

By focusing on the 5 years since the introduction of the Japanese government's "New Robot Strategy", we conducted an online questionnaire survey to investigate the following two research questions:
RQ1: What type of robot is socially accepted?

RQ2: Can experiences with and knowledge of robots affect social acceptance of the robots? Moreover, is this affection different dependent on the types of robots?

The survey focused on three types of robots: vacuum, pet-type, and communication robots. These types of robots are representative of robots that have been introduced into daily life and are commonly seen in the media in Japan. In fact, a survey conducted for 97 Japanese students in the first-year at a university (Nomura and Ura 2018) revealed that their images of real robots consisted of humanoids like Pepper and vacuum robots like Roomba. Moreover, the types of robot Japanese people preferred, revealed by Kochigami et al. (2018), included a pet-type robot. Thus, we investigated the above research questions by comparing these types of robots. This paper presents the results of the analysis of the survey responses and discusses their implications.

\section{Methods}

\subsection{Date and participants}

The survey was conducted in November 2019 through a survey company in Japan. A total of 500 persons, who were randomly selected among people registered with the survey company, were asked to participate in the study. From those aged in their $20 \mathrm{~s}$ to $60 \mathrm{~s}, 50$ males and 50 females in each of the five generation age groups were selected to complete the questionnaire. Table 1 shows the mean age and standard deviation for each generation group. Requests to participate were sent via e-mail, and responses were submitted via a website.

\subsection{Procedure}

The survey was conducted with a within-participant design on types of robots. The survey contained a written explanation of the three types of robots. Just after the explanation of each type of robot, participants were asked to indicate their experiences with each type of robot, their knowledge of the functions of each type of robots, and their degrees of acceptance of each type of robot, including their intention to

Table 1 Mean age and standard deviation for each of the five generation age groups

\begin{tabular}{ll}
\hline Age group & Mean age (SD) \\
\hline $20 \mathrm{~s}(N=100)$ & $26.0(2.9)$ \\
$30 \mathrm{~s}(N=100)$ & $35.5(2.9)$ \\
$40 \mathrm{~s}(N=100)$ & $44.9(2.9)$ \\
$50 \mathrm{~s}(N=100)$ & $54.5(3.0)$ \\
$60 \mathrm{~s}(N=100)$ & $64.4(3.2)$ \\
\hline
\end{tabular}


use it and their level of trust for it. The types of robots were presented in a fixed order; vacuum robots, pet-type robots, and communication robots.

Table 2 shows the explanation of each of the three types of robot. The explanation did not have any visual images such photos and diagrams since there was a possibility that specific visual stimuli could have affected the participants' perception of the robots. We aimed at avoiding this introduced bias by using writing text about the robots' general functions only. These texts included the names of the commercial robots corresponding to the types.

\subsection{Measurements}

Regarding experiences with each type of robot, participants were asked to select one of the following three choices: "(1) I have actually seen the robot", "(2) I have seen the robot only via media such as TV or newspapers", and "(3) I have never seen the robot". Regarding knowledge of the functions of each type of robot, participants were asked to select one of the following four choices: "(1) I know the robot's functions well”, "(2) I know a little about the robot's functions", "(3) I do not know much about the robot's functions", and "(4) I don't know anything about the robot's functions".

For the degree of acceptance of each type of robot, the four items shown in Table 3 were adopted based on Heerink et al.'s study on TAM (Heerink et al. 2010). This measurement consisted of an intention to use, ease of use, likelihood of recommending to others, and trust. Each item had a 5-graded interval.

\section{Results}

\subsection{Coding and reliability of items}

Experiences with and knowledge of robots were reversecoded with higher scores indicating a higher degree of experience and knowledge. The degree of acceptance of robots was also reverse-coded with a higher score indicating higher acceptance.

For each type of robot, Cronbach's reliability coefficient $\alpha$ for the four items related to the degree of acceptance was calculated to confirm the internal consistency. These coefficients were $\alpha=0.921,0.918$, and 0.921 for vacuum, pettype, and communication robots, respectively. Since these values showed sufficient internal consistency, the degree of acceptance of each type of robot was calculated as the sum of the corresponding item scores (min: 4, max: 20).

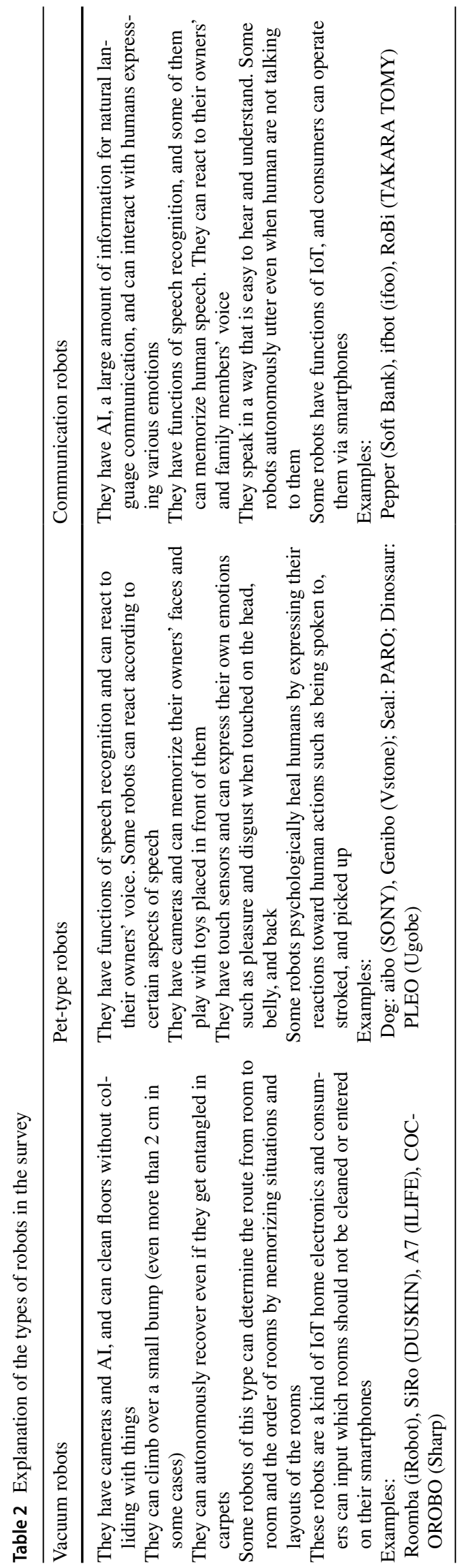


Table 3 Items for measuring degree of acceptance of robots

\begin{tabular}{lll}
\hline Measurement & Item & Choice \\
\hline Intention to use & Would you like to use this type of robot? & 1. Absolutely \\
Ease of use & Do you feel it is easy to use this type of robot? & 2. Yes \\
Recommendation to others & Would you recommend this type of robot to other & 3. Undecided \\
& people? & 4. I don't think so \\
Trust & Can you trust this type of robot? & 5. Absolutely not \\
\hline
\end{tabular}

\subsection{Experiences with robots}

A $\chi^{2}$-test with gender $\times$ experiences $(2 \times 3$ cross table $)$ was conducted for each type of robot. As a result, there was no statistically significant gender bias on experiences with any of the three robot types $\left(\chi^{2}(2)=0.238, p=0.888, V=0.028\right.$ for vacuum robots; $\chi^{2}(2)=1.142, p=0.565, V=0.062$ for pet-type robots; $\chi^{2}(2)=1.853, p=0.396, V=0.079$ for communication robots).

One-way analysis of variance (ANOVA) consisting of experiences with robots as the independent variable and age as the dependent variable was conducted for each type of robot to investigate the relationships between experience and age. There was no statistically significant difference in vacuum robots $\left(F=2.903, p=0.052, \eta^{2}=0.020\right)$. On the other hand, statistically significant differences were found for pet-type and communication robots $(F=4.155$, $p=0.017, \eta^{2}=0.027$ for pet-type robots; $F=7.142$, $p=0.001, \eta^{2}=0.046$ for communication robots). Post-hoc analyses with the Bonferroni method showed that for pettype robots, the mean age of participants who had seen these robots only via media $(M=46.8)$ was higher than the mean age of those who had actually seen these robots $(M=41.7)$ at a statistically significant level of 5\%. Similarly, it was shown that for communication robots, the mean age of participants who had seen these robots only via media $(M=48.0)$ was higher than the mean age of those who had actually seen these robots $(M=47.7)$ and the mean age of those who had never seen these robots $(M=40.8)$ at statistically significant levels of $1 \%$.

Moreover, the Friedman test was conducted to compare experience with the three robot types. The results showed a statistically significant difference between the robot types $\left(\chi^{2}=21.692, p<0.001\right)$. Figure 1 shows the distributions of experiences with each of the three types the robots. Post-hoc analysis based on Wilcoxon tests with Bonferroni correction revealed the tendency that the rate of participants who had actually seen vacuum robots was higher than the rates of those who had actually seen the other types of robots ( $p<0.001, r=0.260$ between vacuum and pet-type robots;

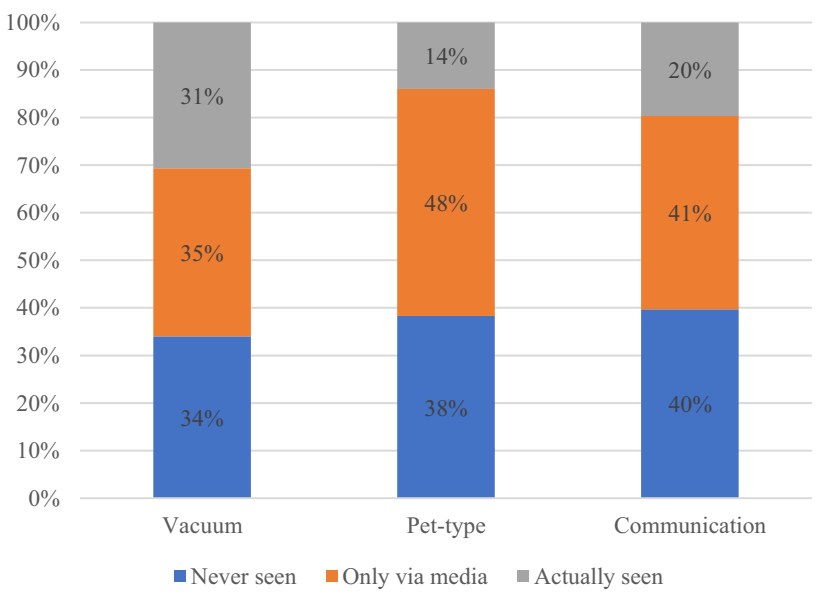

Fig. 1 Distributions of experiences with robots

$p<0.01, r=0.200$ between vacuum and communication robots).

\subsection{Knowledge of functions of robots}

A $\chi^{2}$ test with gender $\times$ knowledge $(2 \times 4$ cross table $)$ was conducted for each type of robot. As a result, there was no statistically significant gender bias on knowledge with any the three robot types $\left(\chi^{2}(3)=0.615, p=0.893, V=0.045\right.$ for vacuum robots; $\chi^{2}(3)=4.922, p=0.178, V=0.128$ for pettype robots; $\chi^{2}(3)=5.025, p=0.170, V=0.129$ for communication robots).

One-way ANOVA consisting of knowledge as the independent variable and age as the dependent variable was conducted for each type of robot to investigate relationships between knowledge and age. There was no statistically significant difference in any of the three types of robots $\left(F=2.035, p=0.109, \eta^{2}=0.020\right.$ for vacuum robots; $F=1.968, p=0.119, \eta^{2}=0.020$ for pet-type robots; $F=2.459, p=0.052, \eta^{2}=0.024$ for communication robots).

Moreover, the Friedman test was conducted to compare knowledge between the robot types. The results showed a statistically significant difference between the robot types $\left(\chi^{2}=14.725, p<0.001\right)$. Figure 2 shows the distributions of knowledge of functions of the robots. Post-hoc analysis based on Wilcoxon tests with Bonferroni correction revealed the tendency that the rates of participants who knew about 


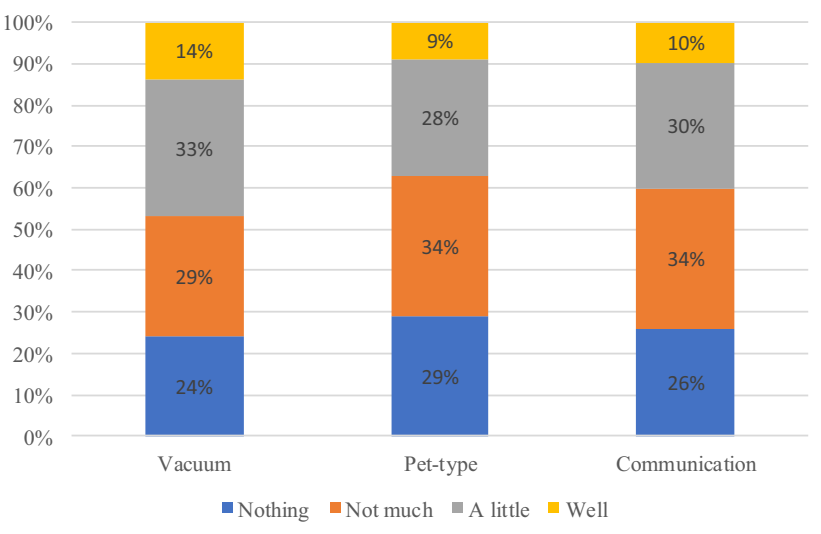

Fig. 2 Distributions of knowledge of functions of robots

the functions of vacuum robots well or a little were higher than the rates of those who knew about the functions of the other types of robots $(p<0.001, r=0.230$ between vacuum and pet-type robots; $p<0.05, r=0.150$ between vacuum and communication robots).

\subsection{Social acceptance of robots}

Three-way mixed ANOVA with gender (male, female) $\times$ generation (five age groups from 20 to $60 \mathrm{~s}$ ) $\times$ robot types (vacuum, pet-type, communication; within-participant factor) was conducted for the acceptance score of the robots to investigate relationships between gender, age, and types of robots. Table 4 shows the results. Only the main effect of robot types was statistically significant.

Post-hoc analysis based on paired $t$ tests with Bonferroni correction revealed statistically significant differences between vacuum and pet-type robots $(t=6.575, p<0.001$, $r=0.360)$, between vacuum and communication robots ( $t=4.372, p<0.001, r=0.250)$, and pet-type and communication robots $(t=-3.286, p<0.01, r=0.190)$. Figure 3 shows the means and standard deviations of the acceptance scores of the robots.

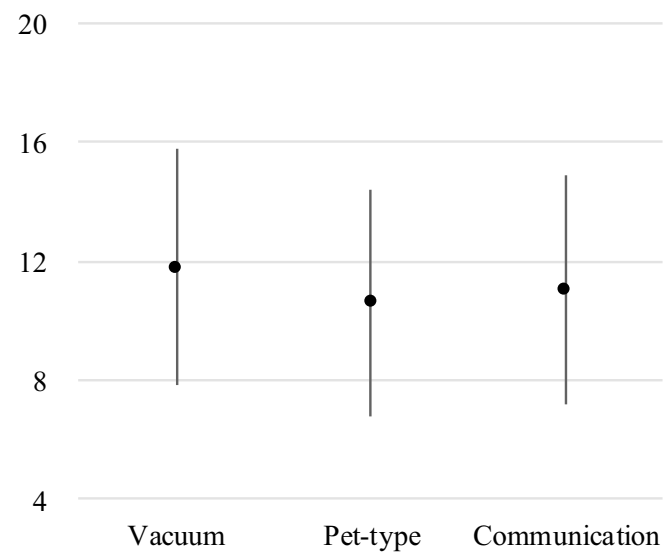

Fig. 3 Means and standard deviations of acceptance scores of robots

\subsection{Influence of experience and knowledge on social acceptance of robots}

Linear regression analyses with experiences with and knowledge of functions of robots as independent variables and acceptance scores of robots as the dependent variable were conducted for each type of robots to investigate the relationships between social acceptance of the robots, and experience and knowledge. Table 5 shows the results.

It was found that in vacuum robots experience and knowledge positively affected the acceptance of the robots, although only the knowledge affected on pet-type and communication robots.

\section{Discussion}

\subsection{Findings and implications}

Gender did not affect experiences with or knowledge of functions of vacuum, pet-type, or communication robots investigated in the survey. Moreover, age did not affect experiences with or knowledge of functions of vacuum robots. On the other hand, the mean age of participants who had
Table 4 Results of three-way mixed ANOVA for acceptance score of robots

\begin{tabular}{|c|c|c|c|c|}
\hline & & $F$ & $p$ & $\eta_{\mathrm{p}}^{2}$ \\
\hline \multirow[t]{3}{*}{ Main effect } & Robot type & 27.282 & $<0.001$ & 0.086 \\
\hline & Gender & 0.217 & 0.642 & 0.001 \\
\hline & Generation & 0.306 & 0.874 & 0.004 \\
\hline \multirow[t]{3}{*}{ First order interaction effect } & Robot type $\times$ gender & 0.539 & 0.584 & 0.002 \\
\hline & Robot type $\times$ generation & 1.448 & 0.173 & 0.020 \\
\hline & Gender $\times$ generation & 0.252 & 0.908 & 0.003 \\
\hline Second order interaction effect & & 1.001 & 0.434 & 0.014 \\
\hline
\end{tabular}


Table 5 Results of linear regression analyses for acceptance scores of robots

\begin{tabular}{|c|c|c|c|c|c|c|c|c|c|}
\hline & \multicolumn{3}{|c|}{ Vacuum robots } & \multicolumn{3}{|c|}{ Pet-type robots } & \multicolumn{3}{|c|}{ Communication robots } \\
\hline & $\beta$ & $t$ & $p$ & $\beta$ & $t$ & $p$ & $\beta$ & $t$ & $p$ \\
\hline Experience & 0.266 & 3.480 & 0.001 & 0.005 & 0.063 & 0.949 & -0.003 & -0.040 & 0.968 \\
\hline Knowledge & 0.208 & 2.722 & 0.007 & 0.287 & 3.596 & 0.000 & 0.381 & 5.098 & 0.000 \\
\hline & Adjusted $R^{2}$ & & 0.189 & Adjusted $R^{2}$ & & 0.078 & Adjusted $R^{2}$ & & 0.138 \\
\hline
\end{tabular}

Bold: independent variables having statistically significant effects seen pet-type and communication robots only via media was higher than that of those who had actually seen these robots or had never seen these robots. This suggests age differences in experiences with robots in Japan depending on the type of robot.

In the results of the survey, there were differences between the three robot types in all measures. First, experiences with and knowledge of functions of vacuum robots were higher than those of pet-type and communication robots. Second, there was a clear order of social acceptance of these robots: vacuum robots $>$ communication robots $>$ pet-type robots. Moreover, knowledge of functions of these robots positively affected social acceptance, although experience influenced acceptance only in the case of vacuum robots.

The above results can be summarized as follows:

1. Vacuum robots were more socially accepted than pettype and communication robots in Japan (RQ1)

2. Experiences with and knowledge of functions of vacuum robots were not dependent on gender or age. Moreover, social acceptance of this type of robot increased as experience and knowledge increased (RQ2)

3. Although experiences with pet-type and communication robots were age-dependent, this did not influence the social acceptance of these robots. Knowledge of functions of these robots, which had neither age nor gender dependency, positively affected social acceptance (RQ2)

The cause of the above results cannot strictly be investigated within this survey. However, we can infer some reasons. The key concept is the ease to assume robots' behaviors. It is hypothesized that humans accept a specific type of robot more positively if its behaviors can be more easily assumed.

The aim and functions of vacuum robots are clearer than those of pet-type and communication robots. In other words, vacuum robots have a single task, i.e., the cleaning of houses, and their functions are clearly aimed at that task. In fact, the instruction texts presented in the survey reflect it. This clarity may mediate between experiences with and knowledge of the functions of this robot type and the ease to assume its behaviors, and, thereby leading to higher social acceptance.
On the other hand, pet-type and communication robots are aimed at abstract tasks, i.e., interaction with humans, regardless of linguistic or non-linguistic style. Thus, simple experiences with these robots may not cause acceptance, and only knowledge of the functions may contribute with the ease to assume their behaviors and acceptance of them. Moreover, it is estimated that communication robots can provide more ease to assume their behaviors via anthropomorphism than pet-type robots (Duffy 2003; Epley et al. 2007; Spatola et al. 2019a). It may lead to the difference in social acceptance between these two robot types.

The above estimation may have an important implication: in instances where a robot's action and functions are clear, the robot may be more socially accepted. This implies that robot design focusing on a specific aim and task may lead to greater social acceptance.

\subsection{Limitations}

The results of this survey suggested differences in social acceptance of robots depending on their type, and the influences of experiences with and knowledge of functions of robots on their social acceptance in Japan 5 years after the national government's “New Robot Strategy”. However, the results are hard to generalize from the following two perspectives. First, our study participants were all Japanese. Thus, this research should be considered a case study focusing on Japan. Second, the participants were recruited via a survey company. Thus, there is a concern about whether they were representative samples in Japan 5 years after "New Robot Strategy".

This study also has some methodological limitations. First, the survey adopted only a written explanation about the three robot types without any concrete visual stimuli such as photos or pictures to avoid influences of specific visual stimuli into the participants' perception of the robots. On the other hand, the description of the robots included some concrete names of robots. Thus, this conflict may have caused individual differences in images of the robots. Second, the types of robots were presented in a fixed order. We cannot deny the possibility that it affected the whole measures. Third, only four items were used to measure the participants' degree of acceptance of the robots, and other psychological constructs 
for concrete types of robots. Thus, detailed measurement of social acceptance of robots, such as that expressed by subscales in the Technology Acceptance Model (Heerink et al. 2010), was not conducted. Fourth, the survey simply adopted the three types of robot for comparison on acceptances, experiences, and knowledge, and did sufficiently not take into account differences on concrete characteristics such as appearances and behaviors. In particular, the problem on how the degrees of anthropomorphism of the robots affected the acceptances, experiences, and knowledge should have been considered because of the complexity of anthropomorphism itself (Fink 2012).

Moreover, the results of the survey did not suggest gender or age influences on the social acceptance of robots. This was not consistent with some existing studies (e.g., (Scopelliti et al. 2005; Nomura 2017)). Thus, it should be investigated what factor can mediate gender and age effects on social acceptance of robots. In addition, although the results of the survey revealed differences between some robot types on their acceptances and relationships with experiences and knowledge in the current stage, it cannot be clarified whether and how these acceptances and experiences changed for the 5 years since the introduction of the Japanese government's "New Robot Strategy".

In a future study, we are planning to extend the sampling of the survey to the United States, European Union, and other Asian nations. Moreover, this future study should include personal traits that were not dealt with in the current study, such as educational backgrounds, occupations, attitudes, and anxiety toward technologies. In addition, we should repeat this survey every few years to investigate changes over time in social acceptance of robots and its relationships with experiences and knowledge of them.

Acknowledgements This research was supported in part by a Grantin-Aid for Scientific Research (No. 20H05573) from the Japan Society for the Promotion of Science.

\section{Declarations}

Conflict of interest The authors declare that they have no conflict of interest.

Open Access This article is licensed under a Creative Commons Attribution 4.0 International License, which permits use, sharing, adaptation, distribution and reproduction in any medium or format, as long as you give appropriate credit to the original author(s) and the source, provide a link to the Creative Commons licence, and indicate if changes were made. The images or other third party material in this article are included in the article's Creative Commons licence, unless indicated otherwise in a credit line to the material. If material is not included in the article's Creative Commons licence and your intended use is not permitted by statutory regulation or exceeds the permitted use, you will need to obtain permission directly from the copyright holder. To view a copy of this licence, visit http://creativecommons.org/licenses/by/4.0/.

\section{References}

Bröhl C, Nelles J, Brandl C, Mertens A, Nitsc V (2019) Human-robot collaboration acceptance model: development and comparison for Germany, Japan, China and the USA. Int J Soc Robot 11:709-726

Davis FD (1989) Perceived usefulness, perceived ease of use, and user acceptance of information technology. MIS Q 13(3):319

De Graaf MM, Allouch SB (2013) Exploring influencing variables for the acceptance of social robots. Robot Auton Syst 61(12):1476-1486

Duffy BR (2003) Anthropomorphism and the social robot. Robot Auton Syst 42(3-4):177-190

Epley N, Waytz A, Cacioppo JT (2007) On seeing human: a threefactor theory of anthropomorphism. Psychol Rev 114(4):864-886

Fink, J. (2012). Anthropomorphism and human likeness in the design of robots and human-robot interaction. In: Proceedings of the International Conference on Social Robotics (ICSR 2021), pp 199-208

Heerink M, Kröse B, Evers V, Wielinga B (2010) Assessing acceptance of assistive social agent technology by older adults: the Almere Model. Int J Soc Robot 2:361-375

Kochigami K, Okada K, Inaba M (2018) Social acceptance of interactive robots in Japan: comparison of children and adults and analysis of people's opinion. In: Companion of the 2018 ACM/ IEEE International Conference on Human-Robot Interaction, pp $157-158$

Latikka R, Turja T, Oksanen A (2019) Self-efficacy and acceptance of robots. Comput Hum Behav 93(4):157-163

Lee H, Kang H, Kim MG, Lee J, Kwak SS (2016) Pepper or roomba? Effective robot design type based on cultural analysis between Korean and Japanese users. Int J Softw Eng Appl 10(8):37-46

Ministry of Internal Affairs and Communication, Japan. (2019). Information and Communications in Japan 2019 (summary). https:// www.soumu.go.jp/main_sosiki/joho_tsusin/eng/whitepaper/2019/ index.html

Morikawa M (2017) Firms' expectations about the impact of AI and robotics: evidence from a survey. Econ Inq 55(2):1054-1063

Nitto H, Taniyama D, Inagaki H (2017) Social acceptance and impact of robots and artificial intelligence: findings of survey in Japan, the US and Germany. NRI Papers No. 211

Nomura T (2014) Influences of experiences of robots into negative attitudes toward robots. In: Proceedings of the 23rd IEEE International Symposium on Robot and Human Interactive Communication (RO-MAN 2014), pp 460-464

Nomura T (2017) Robots and Gender. Gender Genome 1:18-25

Nomura T, Ura K (2018) Exploration of basic images about robots in adolescents: a survey based on drawings in Japan. In: Proceedings of the 6th International Conference on Human-Agent Interaction (HAI 2018), pp 321-323

Nomura T, Suzuki T, Kanda T, Kato K (2006) Measurement of negative attitudes toward robots. Interact Stud 7:437-454

Prime Minister of Japan and His Cabinet (2015) New robot strategy: Japan's robot strategy—vision, strategy, action plan. https://www. kantei.go.jp/jp/singi/keizaisaisei/pdf/robot_honbun_150210EN. pdf

Reich N, Eyssel F (2013) Attitudes towards service robots in domestic environments: the role of personality characteristics, individual interests, and demographic variables. Paladyn J Behav Robot 4(2):123-130

Scopelliti M, Giuliani MV, Fornara F (2005) Robots in a domestic setting: a psychological approach. Univ Access Inf Soc 4:146-155

Smarr C-A, Prakash A, Beer JM, Mitzner TL, Kemp CC, Rogers WA (2012) Older Adults' Preferences for and acceptance of robot assistance for everyday living tasks. In: Proceedings of the Human Factors and Ergonomics Society Annual Meeting 56(1): 153-157 
Spatola N, Monceau S, Ferrand L (2019a) Cognitive impact of social robots: how anthropomorphism boosts performance. IEEE Robot Autom Mag 27(3):73-83

Spatola N, Anier N, Redersdorff S, Ferrand L, Belletier C, Normand A, Huguet P (2019b) National stereotypes and robots' perception: the "made in" effect. Front Robot AI 6:21

Weiss A, Bernhaupt R, Tscheligi M, Yoshida E (2009) Addressing user experience and societal impact in a user study with a humanoid robot. In: Proceedings of the 1st Symposium on New Frontiers in Human-Robot Interaction, pp 150-157

Publisher's Note Springer Nature remains neutral with regard to jurisdictional claims in published maps and institutional affiliations. 